tial medication errors, drug interactions and adverse reactions. \section{appropriateness of prescribing and frailty in elderly patients using STOPP criteria and Frailty Index in tertiary care teaching hospital}

\author{
Sweta Nariya, Ajita Pillai, \\ Sneha Agravat \\ Department of Pharmacology, PDU \\ Govt. Medical College, Rajkot, \\ India
}

\section{Abstract}

People above 65 years of age would amount to $10.7 \%$ of total population of India by 2021 . India has acquired the label of 'an ageing nation' with $7.7 \%$ of its population being more than 60 years old. Geriatric patients are exposed to potentially inappropriate medications (PIMs) thus strategies to improve quality of life and safety of prescribing is needed. One such explicit criteria tool is 'Screening Tool of Older person's Potentially inappropriate Prescriptions' (STOPP) criteria. For older individuals, frailty plays a decisive role. Frailty assessment is useful for identification of risk stratifications to assist in clinical decision makings. Hence, this study is aimed to assess the percentage of PIM using STOPP criteria and correlation between PIMS and Frailty Index (FI) in elderly patients in our setting. An observational study was done in 60 patients in department of medicine at tertiary care teaching hospital. Demographic details, diagnosis and current medications were recorded in Microsoft excel 2016. PIMs based on STOPP criteria version 2 and FI suggested by Searle et al. was calculated and analyzed. Spearman rank correlation test was used to check the correlation between FI and PIM. Out of 60 prescriptions, 21 prescriptions with PIM were found. Most common drugs prescribed as PIM were Aspirin, calcium, ceftriaxone, multivitamin B complex and furosemide. FI was analyzed by using 34 variables. Value of FI was between 0.03 to 0.17 . Spearman Rank correlation test showed direct low degree of significant correlation between FI and PIM with correlation (r) value of 0.1602 (P value $=0.02)$.

$35 \%$ of prescriptions were having at least one PIM in our study and we found that there was direct association between FI and PIM which will help us to reduce poten-

\section{Introduction}

Over the last century, there have been dramatic increases in life expectancy owing largely to improvements in living standards and advances in diagnostics, pharmaceutical medicine and therapeutics. ${ }^{1}$ People above 65 years of age would amount to $10.7 \%$ of total population of India by 2021. India has acquired the label of 'an ageing nation' with $7.7 \%$ of its population being more than 60 years old. ${ }^{2}$ Though increased longevity is to be celebrated, it is well established that increasing age brings with it an increase in the burden of co-morbidity. ${ }^{3}$ Older people often have several coexisting medical problems and take multiple drugs. Increasing numbers of medications is associated with a higher risk of adverse drug events (ADEs) with resultant increased frequency of hospitalization, negative health outcomes and increased healthcare resource utilization.

Geriatric patients are exposed to potentially inappropriate prescriptions (PIP) defined as over use of drugs, irrational choice leading to prescription of a potentially inappropriate medication (PIM) ${ }^{4}$ Various strategies to identify, measure and reduce potentially inappropriate prescribing have been the focus of worldwide research endeavors over the last thirty years. ${ }^{3}$ One such explicit criteria tool is 'Screening Tool of Older person's Potentially inappropriate Prescriptions' (STOPP) criteria.

For older individuals, frailty plays a decisive role in increasing adverse health outcomes in most clinical situations. ${ }^{5}$ Many tools or criteria have been introduced to define frailty in recent years. Frailty Index is one such tool. Frailty assessment is useful for identification of those at higher risk for adverse outcomes and for risk stratifications to assist in clinical decision makings.

There are very few systematic studies evaluating PIM and frailty in elderly patients. Also, there is paucity of Indian studies comparing PIM and frailty index. Present study evaluates the appropriateness of prescribing pattern using STOPP criteria and frailty in our set up.

\section{Aims and objectives}

The aims and objectives of the study were: i) to assess PIM in elderly patients using STOPP criteria and Frailty Index (FI); ii) to assess correlation between PIMs and FI.
Correspondence: Sneha Agravat, Department of Pharmacology, PDU Govt. Medical College, Jamnagar road, Rajkot, India. Tel.: +81.400425684

E-mail: agarwal_sneha85@yahoo.com

Key-words: STOPP criteria; potentially inappropriate medication; Frailty Index.

Acknowledgments: we would like to acknowledge the faculties of medicine department of our institute for their cooperation throughout my study period.

Contributions: SN, concepts, design, data acquisition, data analysis, statistical analysis, manuscript preparation; AP, concepts, design, literature search, manuscript editing, manuscript review; SA, literature search, definition of intellectual content, data analysis, manuscript editing.

Conflict of interest: the authors declare no potential conflict of interest:

Availability of data and materials: from case files and treatment sheets of elderly inpatients by regular visits to IPD (Indoor patients department) of medical ward of tertiary care teaching hospital.

Ethics approval and consent to participate: approval for conduction of study was taken from Institutional ethical committee. PDU/ MCR/IEC/19984/2019. Regd. No. - ECR/ 635/INST/GJ 2014. Prior informed consent has been taken from all study participants before enrolling them in the study.

Received for publication: 7 May 2021.

Revision received: 6 October 2021.

Accepted for publication: 6 October 2021.

This work is licensed under a Creative Commons Attribution-NonCommercial 4.0 International License (CC BY-NC 4.0).

${ }^{\circ}$ Copyright: the Author(s), 2021

Licensee PAGEPress, Italy

Geriatric Care 2021; 7:9845

doi:10.4081/gc.2021.9845

\section{Materials and Methods}

An observational study was carried out in the department of medicine at tertiary care teaching hospital after obtaining approval from Institutional Ethical Committee (IEC). Prescriptions of total 60 patients were included and analyzed in this study.

\section{Inclusion criteria}

The inclusion criteria the study were: i) patients of either sex aged $\geq 65$ years, admitted in medicine department of our tertiary care teaching hospital; ii) only those 
patients who have been diagnosed accurately by Physicians.

All the patients who participated in the study were given clear explanations about the purpose and nature of the study in the language they understood. The study was carried out by regular visits to IPD (Indoor patients department) and information from case sheets of elderly inpatients was collected. Following details of patients were collected and recorded: i) demographic details; ii) clinical and therapeutic data: diagnosis and drug details; iii) co-morbidities and treatment details; iv) drug details-generic name, dose and route of administration, frequency of administration.

STOPP criteria was applied for every drug of the prescription individually and marked as PIM whenever found. Total number of prescriptions with PIMs was analyzed using STOPP criteria version 2. FI is a simple calculation of the presence or absence of each deficit as a proportion of the total deficits. Thus, frailty is defined as the cumulative effect of individual deficits. Total 34 health deficits have been included as a part of FI in our study. Construction of FI was done as suggested by Searle et al. ${ }^{6}$ All the variables collected were assessed for suitability for inclusion in FI. All binary variables were coded as 1 or $0(1=$ presence of deficit, $0=$ absence of deficit). Quartiles were cut at $0.06,0.09$ and 0.14 for FI. Analysis of FI is done in the range of 0.06 to $0.09,0.1$ to 0.14 and $>0.14$.

\section{Statistical analysis}

All data were collated using Microsoft excel 2016. Demographic details, PIMs and FI has been analyzed as percentage using descriptive statistics. Spearman rank correlation test was used to check the correlation between Frailty index and PIM $(\mathrm{P}<0.05)$.

\section{Results}

Out of 60 patients, $45 \%$ were male and $55 \%$ were female. The STOPP criteria identified 21 prescriptions with PIM. As per STOPP criteria, 16 prescriptions have only one PIM, 2 prescriptions have 2 PIMs, and 3 prescriptions were having 3 PIMs.

Majority of prescriptions (31\%) were having more than 2 diseases (Figure 2). Polypharmacy was seen in $49 \%$ of prescriptions (Figure 3). Majority of patients were in age group of 65-75 years (Figure 1). PIM distribution among these patients is shown in Table 1.

In our study, the common medicines prescribed were ceftriaxone, enoxaparin, aspirin, clopidogrel, enalapril, metoprolol, ranitidine, metoclopramide, Isosorbide dinitrate, atorvastatin, insulin and multivitamins. Out of these drugs, based on STOPP criteria, most common drug prescribed as PIM was tablet aspirin $(n=6)$ followed by tablet calcium, injection cef- triaxone, multi vitamin B complex drugs and furosemide (Table 2).

FI is analyzed by using 34 variables. In our study, value of FI is between 0.03 to 0.17 and mean FI value is 0.072 . Frequency distribution of FI is shown in Figure 4. In our study, Quartiles were cut at 0.06-lower quartile, 0.09-middle quartile and 0.14upper quartile. Thus, FI value of 0.06 to 0.09 is having mild frailty $(53 \%), 0.1$ to 0.14 is having moderate frailty $(18 \%)$ and $>0.14$ is having severe frailty $(2 \%)$, as depicted in Figure 4.

Correlation between Frailty index and PIM was done using Spearman's rank correlation test. It showed direct low degree of significant correlation between FI and PIM with correlation (r) value of 0.1602 (Figure 5). $\mathrm{P}$ value is 0.02 .

\section{Discussion}

Prescribing for older patients is complex. ${ }^{7}$ Increasing age is associated with changes in pharmacokinetics and pharmacodynamics, so prescribing in this age group can be problematic. Explicit criteria for appropriate prescribing comprise lists of medications that are known to cause harm in older adults; either through predictable pharmacological or predictable physiological mechanisms. ${ }^{3}$ One such explicit criteria tool is the STOPP criteria.

Table 1. Patient characteristics and prevalence of potentially inappropriate medication use.

\begin{tabular}{|c|c|c|}
\hline Characteristics & Frequency (\%) $\mathrm{N}=60$ & STOPP (number of patients with PIM use) (\%) \\
\hline \multicolumn{3}{|l|}{ Gender } \\
\hline Male & $27(45)$ & $10(37.03)$ \\
\hline Female & $33(55)$ & $09(27.27)$ \\
\hline \multicolumn{3}{|l|}{ Age (years) } \\
\hline $65-75$ & $44(73.33)$ & $17(38.64)$ \\
\hline $76-85$ & $11(18.33)$ & $01(9.09)$ \\
\hline $86-95$ & $04(6.67)$ & $01(25)$ \\
\hline $96-100$ & $01(1.67)$ & $00(0)$ \\
\hline \multicolumn{3}{|l|}{ No. of diseases } \\
\hline 1 & 17 & $06(35.29)$ \\
\hline 2 & $19(19.3)$ & $05(26.31)$ \\
\hline 3 & 15 & $04(26.67)$ \\
\hline$\geq 4$ & 10 & $06(60)$ \\
\hline \multicolumn{3}{|c|}{ No. of medications } \\
\hline $1-4$ & 3 & $00(00)$ \\
\hline $5-9$ & $29(48.3 \%)$ & $09(31.03)$ \\
\hline $10-14$ & 20 & $08(40)$ \\
\hline$\geq 15$ & 04 & $03(75)$ \\
\hline
\end{tabular}

PIM, potentially inappropriate medication. 
The latest version 2 comprises of 80 indicators pertaining primarily to important drug-drug and drug-disease interactions and therapeutic duplication, arranged according to relevant physiological systems for ease of use. ${ }^{8}$

In the present study, out of 60 prescriptions PIM was observed more in males which is comparable to the study done by Nagendra Vishwas et al. ${ }^{7}$ and PIM was observed more common in age group of 65-75 years (Figure 1) with mean age of 73.42 years which is like the study done by Kanagasanthosh et al. ${ }^{9}$

Polypharmacy defined as the regular use of five or more medications, is common among older adults. ${ }^{10}$ It is an area of concern for elderly because of several reasons. Elderly people are at a greater risk for adverse drug reactions (ADRs) because of the metabolic changes and reduced drug clearance associated with ageing; this risk is furthermore exacerbated by increasing the number of drugs used. ${ }^{11}$ Polypharmacy may sometimes lead to 'prescribing cascades.' In our study, polypharmacy (5 to 9 drugs) was seen in $48.3 \%$ and hyper polypharmacy ( $>=10$ drugs) was seen in $40 \%$ of study population (Figure 3 ). Similar polypharmacy values were observed in studies done by Herr et al. ${ }^{12}$ (42.9\%) and Porter et al. ${ }^{10}$ (42.7\%), whereas Alsuwaidan et al. ${ }^{13}$ reported $55.7 \%$ of polypharmacy. Comparative lower value of hyper polypharmacy was seen in studies done by Herr et al. ${ }^{12}(27.4 \%)$ and Porter et al. ${ }^{10}(9.5 \%)$.

The global rate of PIM ranges from
$12 \%$ to $40 \%$ and in our study rate of PIM prescriptions is $35 \%$ which is similar to study done by Gallagher et al. ${ }^{14}$ Among Indian studies, our rate of PIM was higher than the study done by Vishwas et al. ${ }^{7}$ $(13.3 \%)$ and Murthy et al. (21.01\%). ${ }^{8}$ The most common PIM is aspirin $(n=6)$ and comparable results were seen in the studies done by Murthy et al. ${ }^{8}$ and Mathur et al. ${ }^{15}$ In the above studies, the most common diseases found in elderly patients are IHD,
CVA, RHD and angina for which Aspirin is prescribed usually by physicians. On the contrary, Vishwas et al. reported Glibenclamide as most common PIM. ${ }^{7}$ Based on STOPP criteria, there is no evidence of added benefit of aspirin over clopidogrel monotherapy in heart failure patients as secondary stroke prevention.

Total 15 PIMs were prescribed without an evidence-based clinical indication which includes injection ceftriaxone, multi

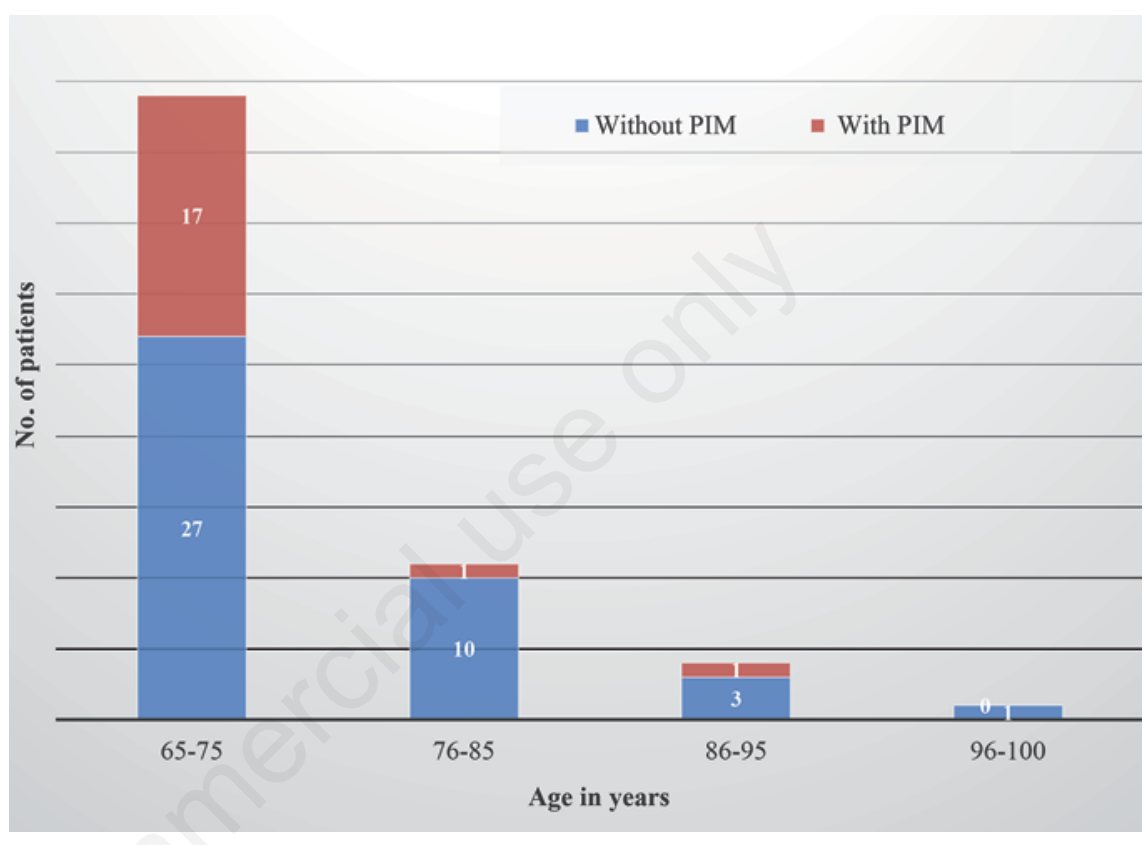

Figure 1. Distribution of potentially inappropriate medication (PIM) use according to age.

Table 2. Classification of potentially inappropriate medication use as per organ system involved identified by sections of STOPP criteria.

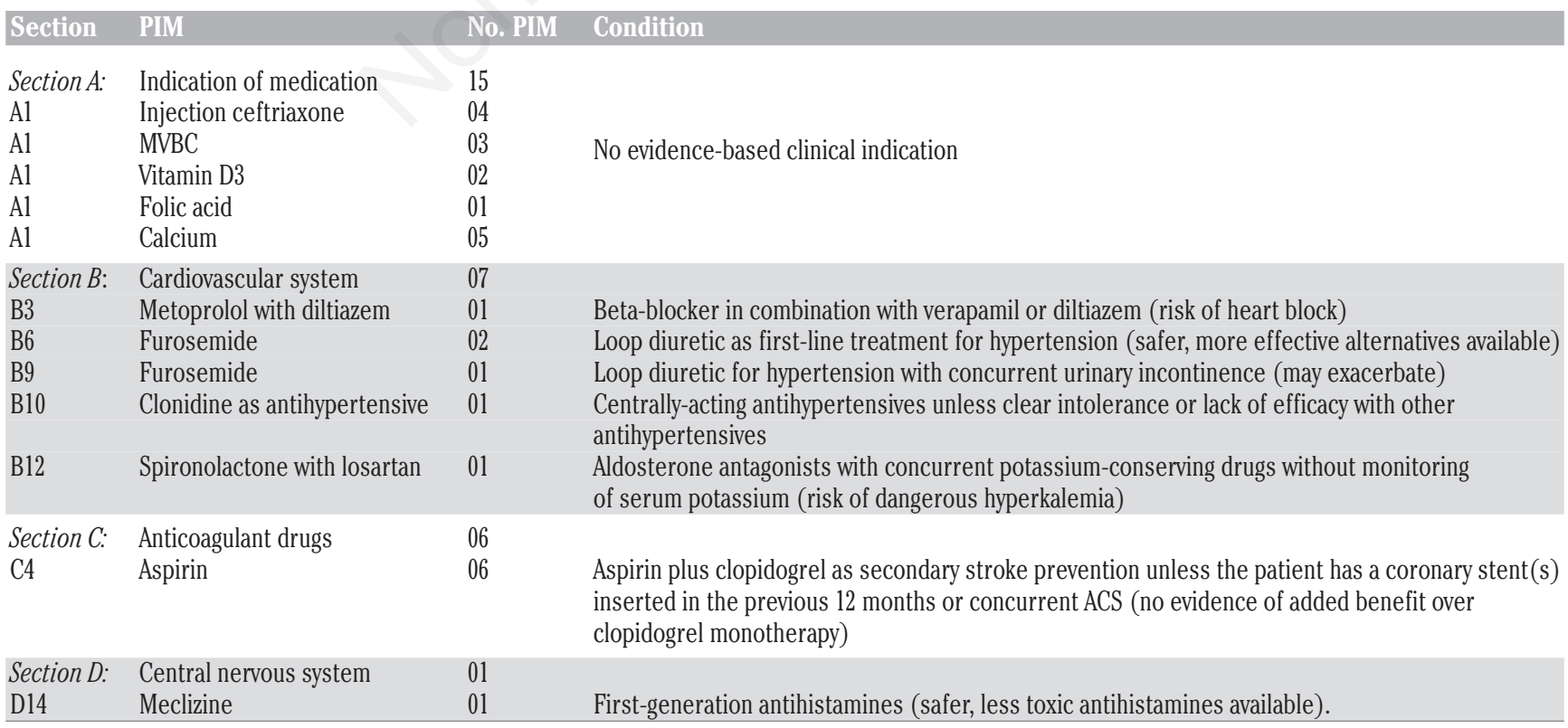

PIM, potentially inappropriate medication; MVBC, multi vitamin B-complex; ACS, acute coronary syndrome. 
vitamin B-complex (MVBC), vitamin D3, folic acid and calcium. Ceftriaxone was prescribed in the case of congestive cardiac failure (CCF), ischemic heart disease (IHD), cerebrovascular accident (CVA) and in the case of glioblastoma with hypertension. Thus, antibiotics were prescribed when not needed and without utilizing the confirmatory laboratory test or evidence based clinical condition. Such practices contribute to the growing problem of antibiotic resistant and antibiotic associated side effects. ${ }^{16}$ In the case of diabetes mellitus and hypertension, MVBC and calcium were prescribed without any evidence based clinical indication. Calcium and vitamin D3 were prescribed in patients with anemia and in patients with uremic encephalopathy without any clinical indications. Loop diuretics was prescribed in 2

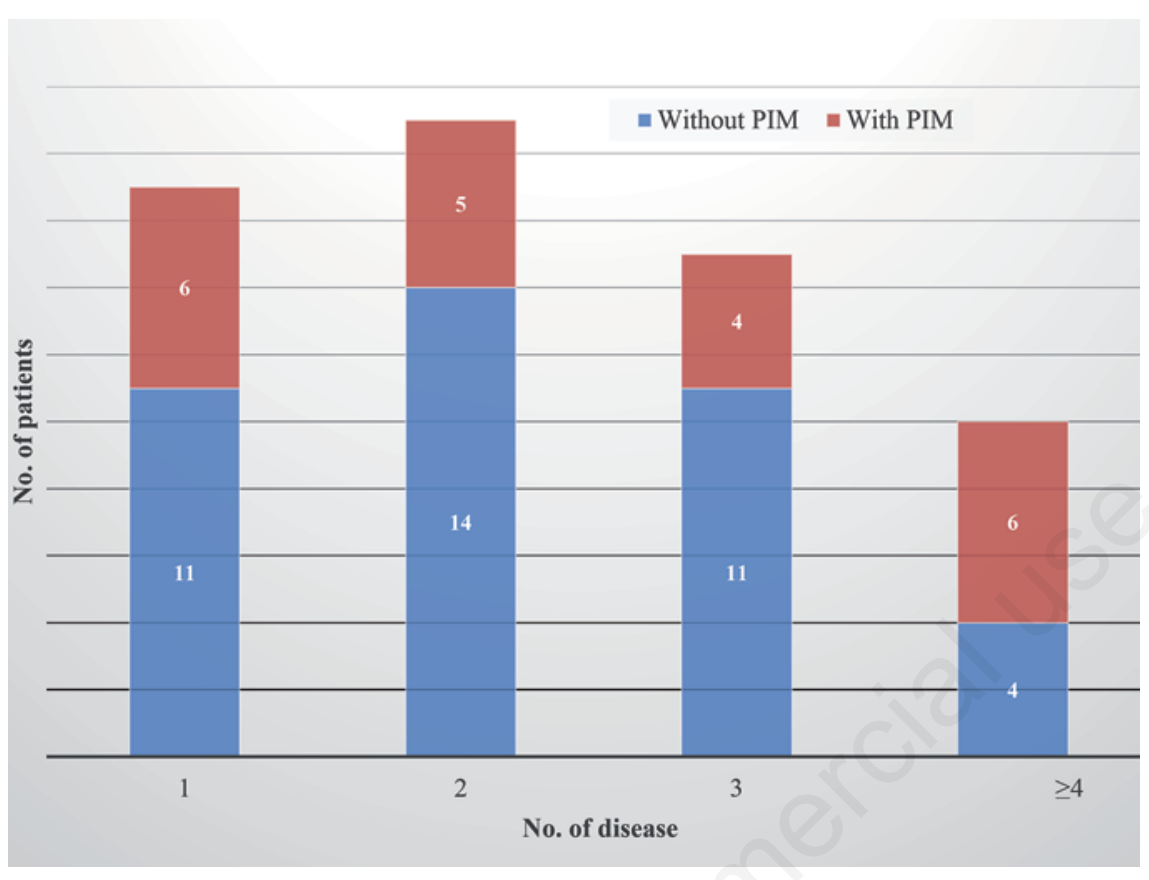

Figure 2. Distribution of potentially inappropriate medication (PIM) use according to No. of disease.

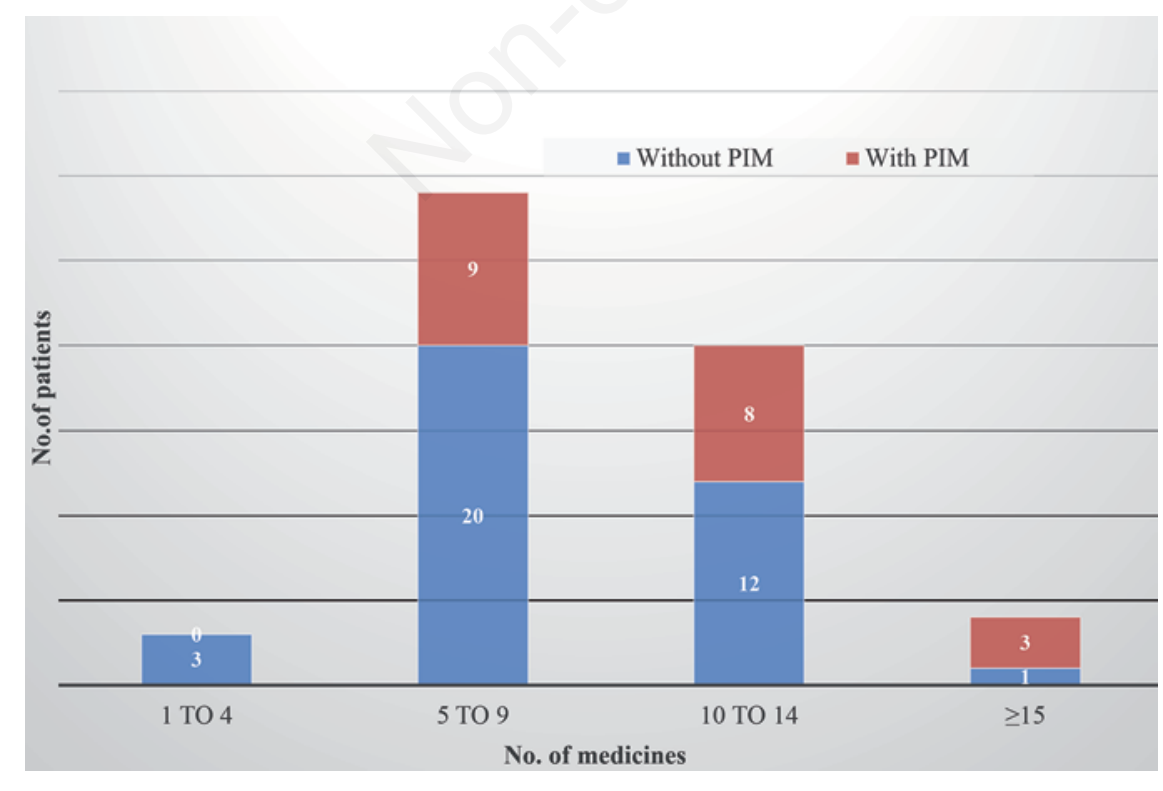

Figure 3. Distribution of potentially inappropriate medication (PIM) according to No. of medicines prescribed. cases of hypertension. It was also prescribed in 1 case of urinary incontinence which may worsen the condition.

In the present study, metoprolol was co-prescribed with diltiazem which increases the risk of heart block. In another case, spironolactone was co-prescribed with losartan without monitoring of serum potassium. Clonidine was prescribed without evidence of any intolerance or lack of efficacy with other antihypertensives. Meclizine was prescribed despite of availability of safer, less toxic antihistamines.

Prescribers should have an appreciation of the potentially low therapeutic yield in very frail older patients with poor life expectancy where the risk of certain treatments can exceed the potential clinical benefit. ${ }^{5}$ Frail older adults often have multi-domain risk factors in terms of physical, psychological, and social health. In the present study, we found that $27 \%$ patients were pre-frail, $53 \%$ had mild frailty, $18 \%$ had moderate frailty and $2 \%$ had severe frailty. On the contrary, study done by Porter et al. ${ }^{10}$ reported $36.4 \%$ of the patients as frail while $45.9 \%$ as prefrail. The likelihood of hospitalization starts to increase with FI of 0.1 and significantly higher above $0.21 .{ }^{17}$ Patients with $\mathrm{FI} \geq 0.16$ are at significantly increased risk of PIP and ADRs. ${ }^{18}$ In our study, 12 patients with frailty index value of $>0.1$ were found.

In our study, there was a low degree of positive and significant correlation between Frailty index and PIM with correlation (r) value of 0.1602 . Similar findings were seen in studies conducted by Khera

et al. (correlation value: 0.280 ) which reported weak positive significant correlation between Frailty index and potentially inappropriate medications (PIM). ${ }^{19}$ It shows that we can decrease the frailty in multimorbid elderly patients by reducing the number of PIMs prescribed to them and direct benefit from this is improvement in quality of life of the elderly patients.

\section{Conclusions}

According to STOPP criteria, 35\% of prescriptions were having at least one PIM in our study and we found that there was direct association between FI and PIM which will help us to reduce potential medication errors, drug interactions and adverse reactions. This could be used as roadmap to take corrective measures and more rational prescribing habit towards this special group of patients. 


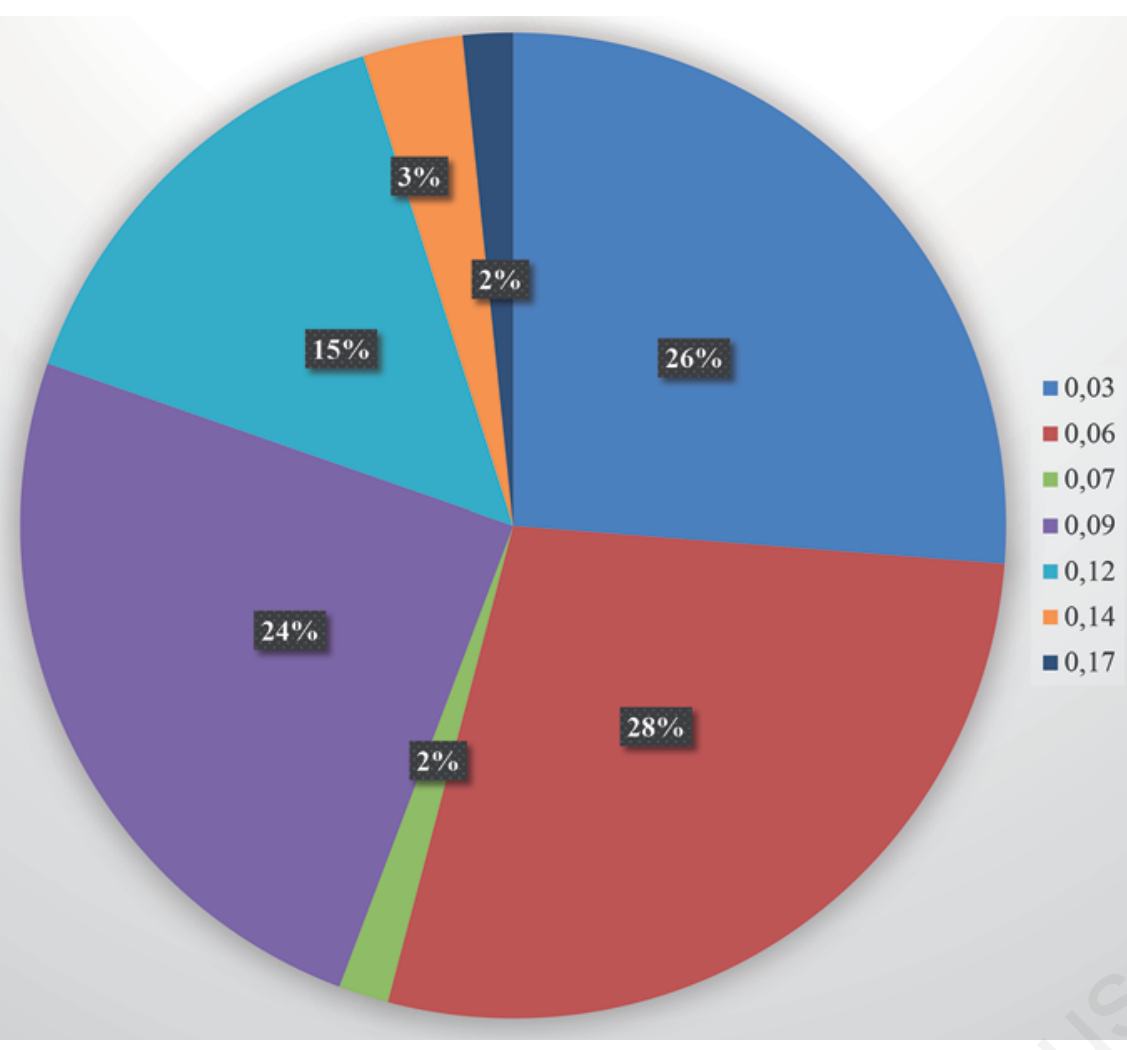

Figure 4. Frequency distribution of Frailty Index.

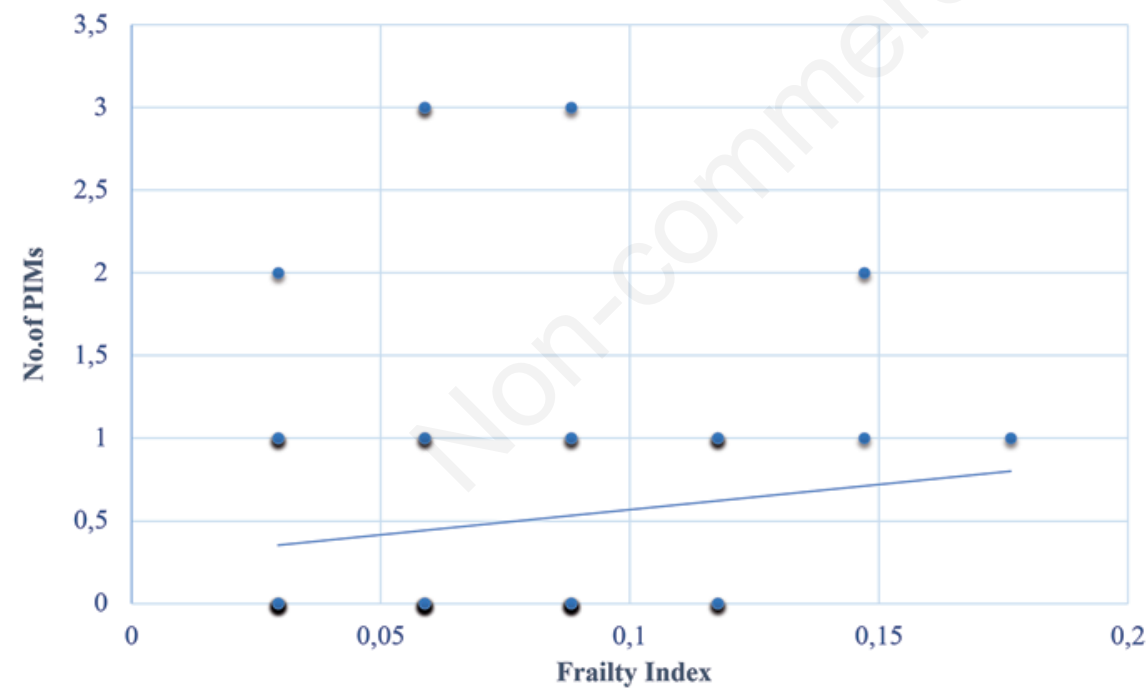

Figure 5. Correlation between potentially inappropriate medications (PIMs) and Frailty Index.

\section{References}

1. Dey S, Nambiar D, Lakshmi JK, Sheikh K, Reddy KS. Health of the elderly in India: challenges of access and affordability. National Academies Press (US); 2012.
2. Ingle GK, Nath A.Geriatric Health in India: concerns and solutions. Indian $\mathrm{J}$ Community Med 2008;33:214-8.

3. Lavan AH, Grady J, Gallagher PF. Appropriate prescribing in the elderly: current perspectives. World J Pharmacol 2015;4:193-209.

4. Moisan J, Baillargeon L, Verreault R, et al. Potentially inappropriate prescriptions for older patients in long-term care. BMC Geriatrics 2004;4:9.

5. Jang I. Frailty and comprehensive geriatric assessment. J Korean Med Sci 2020;35:e16.

6. Searle SD, Mitnitski A, Gahbauer EA, et al. A standard procedure for creating a frailty index. BMC Geriatrics 2008;8-24.

7. Vishwas HN, Anand Harugeri A, Parthasarathi G, Madhan R. Potentially inappropriate medication use in Indian elderly: comparison of Beers' criteria and STOPP criteria. Geriatr Gerontol Int 2012;1-9.

8. Murthy MB, Jagtap K, Burute SR, et al. Geriatric prescription analysis with respect to 'STOPP' and 'START' criteria: a descriptive study in the Indian scenario. Int $\mathrm{J}$ Basic Clin Pharmacol 2017;6:2864-70.

9. Kanagasanthosh K, Topno I, Aravindkumar B. Prevalence of potentially inappropriate medication use and drug utilization pattern in elderly patients: a prospective study from a tertiary care hospital. Int J Res Med Sci 2015;3:2062-72.

10. Porter B, Arthur A, Savva GM. How do potentially inappropriate medications and polypharmacy affect mortality in frail and non-frail cognitively impaired older adults? A cohort study. BMJ Open 2019;9:e026171.

11. Dagli RJ, Sharma A. Polypharmacy: a global risk factor for elderly people. J Int Oral Health 2014;6(6).

12. Herr M, Sirven N, Grondin H, et al. Fraility,polypharmacy and potentially inappropriate medications in old people:findings in a representative sample of the French population. Eur J Clin Pharmacol 2018 [Epub ahead of print].

13. Alsuwaidan A, Almedlej N, Sawsan A, et al. A comprehensive overview of polypharmacy in elderly patients in Saudi Arabia. Geriatrics (Basel) 2019;4:36.

14. Gallagher P, O'Mahony D. STOPP: application to acutely ill elderly patients and comparison with Beers' criteria. Age Ageing 2008;37:673-9.

15. Mathur A, Shah PC. Potentially inappropriate prescribing in elderly: a comparison of Beers and STOPP criteria in tertiary care. Int J Basic Clin Pharmacol 2019;8:95-9.

16. Nathwani D, Dugid M, Brown N, et al. Antimicrobial stewardship from principles to practice. Chapter no. 167. BSAC 2018;13-37:72-7.

17. Clegg A, Young J, Iliffe S, et al. Frailty in elderly people. Lancet 2013;381:752-62.

18. Cullinan S, Mahony D, Sullivan D. Use of a frailty index to identify potentially inappropriate prescribing and adverse drug reaction risks in older patients. Age Ageing 2016;45:115-20.

19. Khera S, Abbasi M, Dabravolskaj J, et al. Appropriateness of medications in older adults living with frailty: impact of a pharmacist-led structured medication review process in primary care. J Prim Care Commun Health 2019;10:1-8. 